\title{
THE BAOUENDI-TREVES APPROXIMATION THEOREM FOR CONTINUOUS VECTOR FIELDS*
}

\author{
SHIFERAW BERHANU ${ }^{\dagger}$ AND JORGE HOUNIE ${ }^{\ddagger}$
}

\begin{abstract}
This article establishes the Baouendi-Treves approximation theorem for locally integrable structures whose vector fields have continuous coefficients. As a consequence, some uniqueness results are derived.
\end{abstract}

Key words. Lowly regular locally integrable systems, Baouendi-Treves approximation theorem, Generic CR submanifolds

AMS subject classifications. Primary 35F15, 35B30, 42B30; Secondary 42A38, 30E25

1. Introduction. Let $\mathcal{M}$ be a $C^{\infty}$ manifold of dimension $N$ and suppose $\mathcal{L}$ is a continuous vector subbundle of $\mathbb{C} T(\mathcal{M})$ with fiber dimension $1 \leq n<N$ and set $m=N-n$. We will say that $\mathcal{L}$ is locally integrable if every $p \in \mathcal{M}$ is contained in an open set $\Omega$ such that there exist $m$ functions $Z_{j}: \Omega \longrightarrow \mathbb{C}, 1 \leq j \leq m$, of class $C^{1}$, satisfying:

(1) If $L$ is a local section of $\mathcal{L}$ defined on $\Omega$, then $L Z_{j}=0,1 \leq j \leq m$;

(2) $d Z_{1}(p) \wedge \cdots \wedge d Z_{m}(p) \neq 0$.

When $\mathcal{L}$ and the first integrals $Z_{1}, \ldots, Z_{m}$ are of class $C^{\infty}$, the approximation theorem of Baouendi and Treves ([BT]) states that every distribution solution $u$ of the sections of $\mathcal{L}$ is locally the limit of a sequence of smooth solutions of the form $P_{k} \circ Z$ where $Z=\left(Z_{1}, \ldots, Z_{m}\right)$ and the $P_{k}$ are holomorphic polynomials. In this latter smooth category, the approximation theorem has had many consequences and has been a tool in dozens of papers, mainly dealing with CR theory (approximation of CR distributions, extension of CR distributions, propagation of analyticity), local behavior of solutions (local solvability, hypoellipticity, boundary behavior of solutions of homogeneous equations, uniqueness in the Cauchy problem) and miscellaneous topics like the similarity principle, the F. and M. Riesz theorem, representation of solutions, etc. To discuss uniqueness results, we recall that a submanifold $\Sigma \subseteq \mathcal{M}$ is called maximally real if for each $p \in \Sigma$,

$$
\mathbb{C} T_{p}(\mathcal{M})=\mathbb{C} T_{p}(\Sigma) \oplus \mathcal{L}_{p}
$$

In the $\mathrm{CR}$ case, that is, when $\mathcal{L} \cap \overline{\mathcal{L}}=0, \Sigma$ is maximally real if and only if it is totally real of maximal dimension. In the smooth case, if $\Sigma$ is a maximally real submanifold and $u$ is a distribution solution, the approximating functions $P_{k} \circ Z$ in the Baouendi-Treves approximation theorem are expressed in terms of the trace of $u$ on $\Sigma$. Consequently, if $u$ vanishes on $\Sigma$, then it vanishes in a neighborhood of $\Sigma$. In particular, if $\mathcal{N} \subseteq \mathcal{M}$ is a noncharacteristic hypersurface and a solution $u$ vanishes on $\mathcal{N}$, then it vanishes in a neighborhood of $\mathcal{N}$ since each $p \in \mathcal{N}$ is contained in a maximally real submanifold $\Sigma \subseteq \mathcal{N}$. The unique determination of a

* Received August 30, 2006; accepted for publication March 13, 2007. Work supported in part by NSF INT-0203005, CNPq and FAPESP.

† Department of Mathematics, Temple University, Philadelphia, PA 19122-6094, USA (berhanu@ temple.edu). br).

‡ Departamento de Matemática, UFSCar, 13.565-905, São Carlos, SP, Brasil (hounie@dm.ufscar. 
solution by its trace on a noncharacteristic hypersurface has the further consequence that the support of a solution propagates along the Sussmann orbits of $\operatorname{Re} \mathcal{L}=\{X$ : $X=\operatorname{Re} L, L$ a smooth section of $\mathcal{L}\}([\mathrm{T}])$. In this paper we explore the validity of the approximation theorem and the uniqueness results stated above for $\mathcal{L}$ that is assumed to be only continuous with $C^{1}$ local first integrals. When $\mathcal{L}$ is only continuous, we consider solutions $u$ that are measures. In the smooth case, whenever $\Sigma$ is a maximally real submanifold and $u$ is a distribution solution, it is well known that $u$ is a smooth function of variables transversal to $\Sigma$ valued in the spaces of distributions on maximally real submanifolds. In particular, the trace of $u$ on $\Sigma$ - a key ingredient in the approximation theorem - is well defined. When $\mathcal{L}$ is assumed to be only continuous and $u$ is a measure solution, we show in section 2 that near each $p \in \mathcal{M}$, there is a nonvanishing continuous function $a$ such that the trace of $a u$ on each noncharacteristic hypersurface $\mathcal{N}$ is well defined. We also show that if the trace of au on $\mathcal{N}$ vanishes, then $u$ vanishes in a neighborhood of $\mathcal{N}$. This implies the propagation of the support of $u$ along the orbits of $\operatorname{Re} \mathcal{L}$. In section 3 we establish the approximation theorem for measure solutions. The results in this article extend some previous work done in [CR] (see Remark 2.6 for more on this).

2. Regularity and uniqueness for measure solutions. Since the problems we study are local, we will assume that $\mathcal{M}$ is an open subset of $\mathbb{R}^{N}$. Consider a continuous vector subbundle $\mathcal{L}$ of $\mathbb{C} T(\mathcal{M})$ with fiber dimension $1 \leq n<N$ and set $m=N-n$.

Definition 2.1. We say that $\mathcal{L}$ is locally integrable if every $p \in \mathcal{M}$ is contained in an open set $\Omega$ such that there exist $m$ functions $Z_{j}: \Omega \longrightarrow \mathbb{C}, 1 \leq j \leq m$, of class $C^{1}$ satisfying:

(1) if $L$ is a local section of $\mathcal{L}$ defined on $\Omega$, then $L Z_{j}=0,1 \leq j \leq m$;

(2) $d Z_{1}(p) \wedge \cdots \wedge d Z_{m}(p) \neq 0$.

The functions $Z_{1}, \ldots, Z_{m}$ are called a complete set of first integrals. Notice that (1) and (2) imply that $d Z_{1}, \ldots, d Z_{m}$ span the orthogonal bundle $\mathcal{L}^{\perp} \subset \mathbb{C} T^{*}\left(\mathbb{R}^{N}\right)$.

Let $p \in \mathcal{M}$. In a neighborhood $\Omega$ of $p$, we may find $C^{1}$ coordinates $x_{1}, \ldots, x_{m}$, $t_{1}, \ldots, t_{n}$ that vanish at $p$ so that in these coordinates, a complete set of first integrals has the form

$$
Z_{j}(x, t)=x_{j}+i \varphi_{j}(x, t), \quad j=1, \ldots, m,
$$

with $\varphi_{j}$ real satisfying $\partial \varphi_{j} / \partial x_{k}(0,0)=0$. By continuity, we may also assume that $\left|\partial \varphi_{j} / \partial x_{k}(0,0)\right|$ remain small on $\Omega$. In particular, we may assume that the square matrix $Z_{x}=\left[\partial Z_{\ell} / \partial x_{k}\right]$ is nonsingular and hence find continuous function $\lambda_{j k}(x, t)$ such that

$$
\frac{\partial Z_{\ell}}{\partial t_{j}}+\sum_{k=1}^{m} \lambda_{j k} \frac{\partial Z_{\ell}}{\partial x_{k}}=0, \quad j=1, \ldots, n, \quad \ell=1, \ldots, m,
$$

which means that the vector fields

$$
\frac{\partial}{\partial t_{j}}+\sum_{k=1}^{m} \lambda_{j k} \frac{\partial}{\partial x_{k}}, \quad j=1, \ldots, n,
$$

are local generators of $\mathcal{L}$ in $\Omega$. It will be convenient to use another set of generators, 
namely,

$$
L_{j}=\operatorname{det} Z_{x} \frac{\partial}{\partial t_{j}}+\operatorname{det} Z_{x} \sum_{k=1}^{m} \lambda_{j k} \frac{\partial}{\partial x_{k}}, \quad j=1, \ldots, n,
$$

which have the advantage of being antisymmetric, that is,

$$
\int\left(L_{j} u\right) v d x d t=-\int u L_{j} v d x d t, \quad j=1, \ldots, m, \quad u, v \in C_{c}^{\infty}(\Omega),
$$

and may be written as partial differential operators in divergence form

$$
L_{j} u=\frac{\partial\left(\operatorname{det} Z_{x} u\right)}{\partial t_{j}}+\sum_{k=1}^{m} \frac{\partial\left(\operatorname{det} Z_{x} \lambda_{j k} u\right)}{\partial x_{k}}, \quad u \in C_{c}^{\infty}(\Omega) .
$$

Thus, denoting by $\langle u, v\rangle=\int u v d x d t$ the usual duality pairing, if $u \in \mathcal{D}_{0}^{\prime}(\Omega)$, we have that $L_{j} u$ may be defined by

$$
\left\langle L_{j} u, v\right\rangle=-\left\langle u, L_{j} v\right\rangle, \quad v \in C_{c}^{1}(\Omega),
$$

and $L_{j} u \in \mathcal{D}_{1}^{\prime}(\Omega)$. The fact that $u$ satisfies the set of equations

$$
L_{j} u=0, \quad j=1, \ldots, n,
$$

has the following interpretation. Since we are interested in local questions, we may identify top forms $\psi(x) d x_{1} \wedge \cdots \wedge d x_{N}$ with functions $\psi(x)$, view a measure as acting continuously on top forms with continuous coefficients, and state

Definition 2.2. We say the measure $u$ is a solution of $\mathcal{L} u=0$ if for each $p$ and some complete set of $C^{1}$ first integrals $Z_{1}, \ldots, Z_{m}$ near $p$,

$$
\left\langle u, d Z_{1} \wedge \cdots \wedge d Z_{m} \wedge d \omega\right\rangle=0
$$

for any $\omega$ which is an $(n-1)$-form of class $C^{1}$ with compact support.

If we take

$$
\omega=v d t_{1} \wedge \cdots \wedge \widehat{d t}_{j} \wedge \cdots \wedge d t_{n}, \quad v \in C_{c}^{1}(\Omega),
$$

where the hat means that the factor $d t_{j}$ has been omitted, and express $d v$ in the basis $d t_{1}, \ldots, d t_{n}, d Z_{1}, \ldots, d Z_{m}$, we see that

$$
\begin{aligned}
d Z_{1} \wedge \cdots \wedge d Z_{m} \wedge d \omega & =(-1)^{j-1}\left(\operatorname{det} Z_{x}\right)^{-1} L_{j} v d Z_{1} \wedge \cdots \wedge d Z_{m} \wedge d t_{1} \wedge \cdots \wedge d t_{n} \\
& =(-1)^{j-1} L_{j} v d x_{1} \wedge \cdots \wedge d x_{m} \wedge d t_{1} \wedge \cdots \wedge d t_{n}
\end{aligned}
$$

Thus, using (2.4) for $j=1, \ldots, n$, it is easy to check that (2.2) and (2.3) are equivalent conditions for $u \in \mathcal{D}_{0}^{\prime}(\Omega)$ in a neighborhood of $p$. Furthermore, we note that if $(2.3)$ holds for some $\left\{Z_{1}, \ldots, Z_{m}\right\}$, then it also holds for any other complete set of $C^{1}$ first integrals $\left\{W_{1}, \ldots, W_{m}\right\}$. Indeed, by the approximation theorem (which is valid for $C^{1}$ functions) there exist holomorphic functions $F_{k}^{j}$ such that for each $j=1, \ldots, n$, we may write $W_{j}=\lim _{k \rightarrow \infty} F_{k}^{j}\left(Z_{1}, \ldots, Z_{m}\right)$ in the $C^{1}$ topology and so $d W_{1} \wedge \cdots \wedge d W_{m}=$ $\lambda d Z_{1} \wedge \cdots \wedge d Z_{m}$ for some continuous function $\lambda=\lim _{k \rightarrow \infty} H_{k}\left(Z_{1}, \ldots, Z_{m}\right)$ with $H_{k}$ holomorphic. Since this process can be reversed, expressing $d Z_{1} \wedge \cdots \wedge d Z_{m}$ as a multiple of $d W_{1} \wedge \cdots \wedge d W_{m}$, it follows that $\lambda \neq 0$ on a neighborhood of $p$. Observe 
next that, since $H_{k}$ is holomorphic, for any $(n-1)$-form of class $C^{1}$ with compact support $\omega$,

$$
H_{k}(Z) d Z_{1} \wedge \cdots \wedge d Z_{m} \wedge d \omega=d Z_{1} \wedge \cdots \wedge d Z_{m} \wedge d\left(H_{k}(Z) \omega\right)
$$

and therefore,

$$
\begin{aligned}
\left\langle u, d W_{1} \wedge \cdots \wedge d W_{m} \wedge d \omega\right\rangle & =\lim _{k \rightarrow \infty}\left\langle u, H_{k}(Z) d Z_{1} \wedge \cdots \wedge d Z_{m} \wedge d \omega\right\rangle \\
& =\lim _{k \rightarrow \infty}\left\langle u, d Z_{1} \wedge \cdots \wedge d Z_{m} \wedge d\left(H_{k}(Z) \omega\right)\right\rangle=0 .
\end{aligned}
$$

Hence $\left\langle u, d W_{1} \wedge \cdots \wedge d W_{m} \wedge d \omega\right\rangle=0$ for all $(n-1)$-forms $\omega$ of class $C^{1}$ with compact support in a convenient neighborhood of $p$. Assume now that $n=1$, so $\mathcal{L}$ is locally spanned by a single vector field

$$
L=\operatorname{det} Z_{x} \frac{\partial}{\partial t}+\operatorname{det} Z_{x} \sum_{k=1}^{m} \lambda_{k} \frac{\partial}{\partial x_{k}}
$$

and let $u \in \mathcal{D}_{0}^{\prime}(\Omega)$ satisfy the equation

$$
L u=0
$$

in the sense given by (2.3), so $\langle u, L v\rangle=0, v \in C_{c}^{\infty}(\Omega)$. We will prove that $Z_{x}(x, t) u$ may be identified with a function of $t$ valued in $\mathcal{D}^{\prime}\left(Q_{R}\right)$ for a convenient ball $Q_{R} \subset \mathbb{R}^{m}$. Since the statement is local it will be enough to restrict our attention to a neighborhood of the form $\Omega_{R}=Q_{R} \times I_{R}$, where $I_{R}$ is the interval $(-R, R)$ and $Q_{R}$ denotes a ball of radius $R$ centered at the origin of $\mathbb{R}^{m}$. Given $\psi(x) \in C_{c}^{\infty}\left(Q_{R}\right)$, consider the distribution $u_{\psi} \in \mathcal{D}^{\prime}(\mathbb{R})$ defined by

$$
\left\langle u_{\psi}, \phi\right\rangle=\langle u, \psi \otimes \phi\rangle, \quad \phi \in C_{c}^{\infty}\left(I_{R}\right),
$$

where $(\psi \otimes \phi)(x, t)=\psi(x) \phi(t)$. We first point out that $u_{\psi} \in \mathcal{D}_{0}^{\prime}\left(I_{R}\right)$ for $\psi$ fixed. Indeed, writing $U=u_{\psi}$,

$$
|\langle U, \phi\rangle|=|\langle u, \psi \otimes \phi\rangle| \leq C\|\psi \otimes \phi\|_{C^{0}} \leq C^{\prime}(R, \psi)\|\phi\|_{C^{0}}
$$

showing that $U \in \mathcal{D}_{0}^{\prime}\left(I_{R}\right)$. Set

$$
V=\left(\operatorname{det} Z_{x} u\right)_{\psi} \quad \text { and } \quad W=-\sum_{k=1}^{m}\left(\operatorname{det} Z_{x} \lambda_{k} u\right)_{\psi_{x_{j}}} .
$$

Reasoning as before, we conclude that $V$ and $W \in \mathcal{D}_{0}^{\prime}(\mathbb{R})$; furthermore (2.5) implies that

$$
\frac{d V}{d t}=W
$$

holds in the sense of distributions. Thus, there exists a unique function $f_{\psi}(t), t \in \mathbb{R}$, continuous from the right and, after shrinking $I_{R}$ if necessary, of bounded variation on $I_{R}$, such that

$$
\langle V, \phi\rangle=\int f_{\psi}(t) \phi(t) d t .
$$

Since $\psi \mapsto f_{\psi}$ is linear and continuous in $C_{c}^{1}\left(I_{R}\right)$, we may write $f_{\psi}(t)=\langle f(t), \psi\rangle$ and consider $f(t)$ as an element of $\mathcal{D}_{1}^{\prime}\left(Q_{R}\right)$ for each fixed $t$. If $\psi$ belongs to a bounded 
subset $\mathcal{B}$ of $C_{c}^{1}(\mathbb{R})$ and $|t| \leq R$, the variation of $f_{\psi}(t)$ is bounded by a constant depending only on $\mathcal{B}$ and $R$. Thus

$$
\left\langle\operatorname{det} Z_{x} u, \phi \otimes \psi\right\rangle=\int\langle f(t), \psi\rangle \phi(t) d t, \quad \phi(t) \in C_{c}^{\infty}\left(I_{R}\right), \psi(x) \in C_{c}^{\infty}\left(Q_{R}\right) .
$$

More generally, we have that

$$
\left\langle\operatorname{det} Z_{x} u, \phi\right\rangle=\int\langle f(t), \phi(\cdot, t)\rangle d t, \quad \phi(x, t) \in C_{c}^{\infty}\left(\Omega_{R}\right) .
$$

Next for $\phi(x, t)$ a $C^{1}$ function we wish to understand the Leibnitz rule for

$$
\frac{d}{d t}\langle f(t), \phi(\cdot, t)\rangle \text {. }
$$

First fix $\psi(x) \in C_{c}^{1}\left(\mathbb{R}^{m}\right)$. Then we know that in the weak sense (which is what we will always mean)

$$
\frac{d}{d t}\langle f(t), \psi(\cdot)\rangle=W_{\psi}(t)
$$

where for $\varphi(t) \in C_{c}^{0}(\mathbb{R})$,

$$
\left\langle W_{\psi}, \varphi(t)\right\rangle=\sum_{k} \iint\left(\lambda_{k} \operatorname{det} Z_{x} u\right) \frac{\partial \psi}{\partial x_{k}}(x) \varphi(t) d x d t .
$$

We also know that there exists $C>0$ such that $\forall \psi(x) \in C_{c}^{1}\left(B_{r}\right)$, and $|t| \leq R$,

$$
|\langle f(t), \psi\rangle| \leq C|| \psi \|_{C^{1}} .
$$

Let $\phi(x, t) \in C_{c}^{1}$. Consider $F(t)=\langle f(t), \phi(\cdot, t)\rangle$. We will show that $F(t)$ is a function of bounded variation and write a formula for its weak derivative. Clearly, $F(t)$ is a measurable function. From (2.6) we see that $F(t)$ is a bounded function and so it defines a distribution. Suppose now $\phi(x, t)=\psi(x) \phi(t) \in C_{c}^{1}$. Then

$$
\frac{d}{d t}\langle f(t), \phi(\cdot, t)\rangle=\phi^{\prime}(t)\langle f(t), \psi(\cdot)\rangle+\phi(t) W_{\psi}
$$

and so for any $h(t) \in C_{c}^{0}$,

$$
\begin{aligned}
& \left(\frac{d}{d t}\langle f(t), \phi(\cdot, t)\rangle\right)(h)=\iint \operatorname{det} Z_{x} u \psi(x) \phi^{\prime}(t) h(t) d x d t \\
& +\sum_{k=1}^{m} \iint\left(\lambda_{k} \operatorname{det} Z_{x} u\right) \frac{\partial \psi}{\partial x_{k}}(x) \phi(t) h(t) d x d t .
\end{aligned}
$$

More generally, we claim that for any $\phi(x, t) \in C_{c}^{1}$, the formula

$$
\frac{d}{d t}\langle f(t), \phi(\cdot, t)\rangle=\left(\operatorname{det} Z_{x} u\right)_{\phi_{t}}+\sum_{k=1}^{m}\left(\lambda_{k} \operatorname{det} Z_{x} u\right)_{\phi_{x_{k}}}
$$

holds where in the right hand side, $\left(\operatorname{det} Z_{x} u\right)_{\phi_{t}}$ is the distribution in $t$ space defined by

$$
\left\langle\left(\operatorname{det} Z_{x} u\right)_{\phi_{t}}, h(t)\right\rangle=\iint\left(\operatorname{det} Z_{x} u\right)(x, t) \phi_{t}(x, t) h(t) d x d t
$$


and likewise for $\sum_{k}\left(\lambda_{k} \operatorname{det} Z_{x} u\right)_{\phi_{x_{k}}}$. Equation (2.7) shows that (2.8) easily holds for $C^{1}$ functions of the form

$$
\sum_{j=1}^{N} \sum_{k=1}^{M} \psi_{j}(x) \phi_{k}(t) .
$$

The general case follows by approximating a given $C^{1}$ function $\phi(x, t)$ by such finite sums and observing that each side of (2.8) is a continuous operator on the space of $C^{1}$ functions. Observe that (2.8) in particular shows that when $\phi(x, t)$ is a $C^{1}$ function, $\frac{d}{d t}\langle f(t), \phi(\cdot, t)\rangle$ is a measure and hence $\langle f(t), \phi(\cdot, t)\rangle$ is a function of bounded variation.

Recall that for every $t_{0} \in \mathbb{R},\left|t_{0}\right|<R$, $\operatorname{det} Z_{x} u$ has a trace at $t=t_{0}$ which is a distribution of order one. This trace is given by $f\left(t_{0}\right)$ and satisfies the onesided continuity property $\lim _{\varepsilon \backslash 0} f\left(t_{0}+\varepsilon\right)=f\left(t_{0}\right)$. We may also denote this trace by $Z_{x}\left(\cdot, t_{0}\right) u\left(\cdot, t_{0}\right)$ or $Z_{x}\left(x, t_{0}\right) u\left(x, t_{0}\right)$ rather than $f\left(t_{0}\right)$. We now recall the basic operators in the Baouendi-Treves approximation formula, namely

$$
\begin{aligned}
& E_{\tau} u(x, t)=(\tau / \pi)^{m / 2} \int_{\mathbb{R}^{m}} e^{-\tau\left[Z(x, t)-Z\left(x^{\prime}, 0\right)\right]^{2}} \operatorname{det} Z_{x}\left(x^{\prime}, 0\right) u\left(x^{\prime}, 0\right) h\left(x^{\prime}\right) d x^{\prime}, \\
& G_{\tau} u(x, t)=(\tau / \pi)^{m / 2} \int_{\mathbb{R}^{m}} e^{-\tau\left[Z(x, t)-Z\left(x^{\prime}, t\right)\right]^{2}} \operatorname{det} Z_{x}\left(x^{\prime}, t\right) u\left(x^{\prime}, t\right) h\left(x^{\prime}\right) d x^{\prime}, \\
& R_{\tau} u(x, t)=G_{\tau} u(x, t)-E_{\tau} u(x, t),
\end{aligned}
$$

where the notation $\left[Z(x, t)-Z\left(x^{\prime}, t^{\prime}\right)\right]^{2}$ means $\sum_{j=1}^{m}\left(Z_{j}(x, t)-Z_{j}\left(x^{\prime}, t^{\prime}\right)\right)^{2}$ and the function $h(x) \in C_{c}^{\infty}\left(Q_{R}\right)$ is supported in a conveniently small ball and it is identically equal to 1 on a neighborhood of the origin. In the classical case, when $L$ and $Z(x, t)$ are smooth and $u$ is a distribution solution of $L u=0$, it can be proved that $R_{\tau} u(x, t) \rightarrow 0$ as $\tau \rightarrow \infty$ on a small neighborhood of the origin, with convergence in the $C^{\infty}$ topology. We now adapt some of the classical arguments to the present situation, where $u \in \mathcal{D}_{0}^{\prime}$ and $\mathcal{L}$ is of class $C^{0}$, to show that $R_{\tau} u(x, t) \rightarrow 0$ uniformly as $\tau \rightarrow \infty$ on some neighborhood of the origin. Write, for $\zeta \in \mathbb{C}^{m}$ and $t \in I_{R}$,

$$
\begin{aligned}
\tilde{G}_{\tau} u(\zeta, t) & =\left\langle f(t), e^{-\tau[\zeta-Z(\cdot, t)]^{2}} h(\cdot)\right\rangle=\left\langle\operatorname{det} Z_{x}(\cdot, t) u(\cdot, t), e^{-\tau[\zeta-Z(\cdot, t)]^{2}} h(\cdot)\right\rangle \\
& =\int_{\mathbb{R}^{m}} e^{-\tau\left[\zeta-Z\left(x^{\prime}, t\right)\right]^{2}} \operatorname{det} Z_{x}\left(x^{\prime}, t\right) u\left(x^{\prime}, t\right) h\left(x^{\prime}\right) d x^{\prime},
\end{aligned}
$$

and

$$
\tilde{R}_{\tau} u(\zeta, t)=\tilde{G}_{\tau} u(\zeta, t)-\tilde{G}_{\tau} u(\zeta, 0),
$$

so we have

$$
R_{\tau} u(x, t)=(\tau / \pi)^{m / 2} \tilde{R}_{\tau} u(Z(x, t), t) .
$$

If $F: I_{R} \longrightarrow \mathbb{R}$ is a function of bounded variation with weak derivative $F^{\prime}=\mu$, the fundamental theorem of calculus for $F$ states that

$$
\mu((a, b])=\int_{(a, b]} d \mu=F(b+)-F(a+), \quad-R<a<b<R .
$$

Let $F(t)=\tilde{G}_{\tau} u(\zeta, t)$. Since the function $e^{-\tau[\zeta-Z(x, t)]^{2}} h(x)$ is $C^{1}$, we have seen that $F(t)$ is a function of bounded variation, continuous from the right, and formula (2.8) 
leads to

$$
\begin{aligned}
\tilde{R}_{\tau} u(\zeta, t) & =F(t)-F(0) \\
= & \sum_{k=1}^{m} \int_{0}^{t} \int_{\mathbb{R}^{m}} \lambda_{k}\left(x^{\prime}, t^{\prime}\right) \operatorname{det} Z_{x} u\left(x^{\prime}, t^{\prime}\right) \partial_{x_{k}^{\prime}}\left(h\left(x^{\prime}\right) e^{-\tau\left[\zeta-Z\left(x^{\prime}, t^{\prime}\right)\right]^{2}}\right) d x^{\prime} d t^{\prime} \\
& \quad+\int_{0}^{t} \int_{\mathbb{R}^{m}}\left(\operatorname{det} Z_{x^{\prime}} u\right)\left(x^{\prime}, t^{\prime}\right) h\left(x^{\prime}\right) \partial_{t^{\prime}}\left(e^{-\tau\left[\zeta-Z\left(x^{\prime}, t^{\prime}\right]^{2}\right.}\right) d x^{\prime} d t^{\prime}
\end{aligned}
$$

where $\int_{0}^{t}$ must be understood as $\int_{(0, t]}$ for $t \geq 0$ and as $-\int_{(t, 0]}$ for $t<0$. Using the fact that

$$
\left(\partial_{t^{\prime}}+\sum_{k=1}^{m} \lambda_{k} \partial_{x_{k}^{\prime}}\right) e^{-\tau\left[\zeta-Z\left(x^{\prime}, t^{\prime}\right)\right]^{2}}=0
$$

we get

$$
\tilde{R}_{\tau} u(\zeta, t)=\int_{0}^{t} \int_{\mathbb{R}^{m}} u\left(x^{\prime}, t^{\prime}\right) e^{-\tau\left[\zeta-Z\left(x^{\prime}, t^{\prime}\right)\right]^{2}} \operatorname{Lh}\left(x^{\prime}, t^{\prime}\right) d x^{\prime} d t^{\prime}
$$

which, for $\zeta=Z(x, t)$ gives

$$
R_{\tau} u(x, t)=(\tau / \pi)^{m / 2} \int_{0}^{t} d t^{\prime} \int_{\mathbb{R}^{m}} e^{-\tau\left[Z(x, t)-Z\left(x^{\prime}, t^{\prime}\right)\right]^{2}} u\left(x^{\prime}, t^{\prime}\right) L h\left(x^{\prime}, t^{\prime}\right) d x^{\prime},
$$

which extends the classical expression for $R_{\tau} u$. If $\delta>0$ is chosen so that $h\left(x^{\prime}\right) \equiv 1$ for $\left|x^{\prime}\right| \leq 2 \delta$, and we take $|x| \leq \delta$ and $t$ sufficiently small, the exponential in the expression of $R_{\tau} u(x, t)$ can be majorized by $e^{-c \tau}$ for some $c>0$, allowing us to conclude that the estimate $\left|R_{\tau} u(x, t)\right| \leq C e^{-c \tau}$ holds in a neighborhood $\Omega$ of the origin and, in particular, $R_{\tau} u(x, t) \rightarrow 0$ uniformly on $\Omega$ as $\tau \rightarrow \infty$. On the other hand, standard arguments show that, $G_{\tau} u(x, t)$ converges weakly to the measure $u$. It follows that $E_{\tau} u(x, t) \rightarrow u(x, t)$ in $\mathcal{D}_{0}^{\prime}(\Omega)$ as $\tau \rightarrow \infty$. If we assume now that the trace $\operatorname{det} Z_{x}(x, 0) u(x, 0) \equiv 0$, we see that $E_{\tau} u(x, t) \equiv 0$ showing that $u=0$ on $\Omega$. Suppose next the left hand $\operatorname{limit}_{\lim _{t \rightarrow 0^{-}}} Z_{x}(x, t) u(x, t)=\left(Z_{x} u\right)(x, 0-)=0$. Then the equation $\mu([b, 0))=F(0-)-F(b-)$ for $b<0$ implies that $\left(Z_{x} u\right)(x, b-)=0$ for $b<0$. Since $F$ is continuous except possibly on a countable set, we can get $b<0$ arbitrarily close to 0 where $F$ is continuous and hence where the trace $Z_{x}(x, b) u(x, b)=0$. We can then use the approximation scheme as above by using the trace at such $b$ (with $b$ sufficiently small) in the definition of the operators $E_{\tau} u(x, t)$ to conclude that $u(x, t) \equiv 0$ in a neighborhood of the origin. This shows that a function of bounded variation $f(t)$ satisfying

$$
\left\langle\operatorname{det} Z_{x} u, \phi \otimes \psi\right\rangle=\int\langle f(t), \psi\rangle \phi(t) d t, \quad \phi(t) \in C_{c}^{\infty}\left(I_{R}\right), \psi(x) \in C_{c}^{\infty}\left(Q_{R}\right),
$$

cannot have a jump at a point $t=t_{0}$ unless both $f\left(t_{0}+\right) \neq 0$ and $f\left(t_{0}-\right) \neq 0$. Notice that the value $f(t)$ is completely determined outside the at most countable set at which jumps occur and that at the jumps the value of $f$ is arbitrary, unless one imposes, say, that $f$ is continuous from the right or continuous from the left. We may now state and prove 
THEOREM 2.3. Let $\mathcal{L}$ be a locally integrable continuous subbundle of $\mathbb{C} T(\mathcal{M})$. Let $u \in \mathcal{D}_{0}^{\prime}$ be a Radon measure such that $\mathcal{L} u=0$, and let $\Sigma$ be a $C^{1}$ hypersurface noncharacteristic with respect to $\mathcal{L}$. Let $p \in \Sigma$ and let $N=N(p)$ be a unit vector normal to $\Sigma$ at $p$. There exists a nonvanishing continuous function $D$ defined in a neighborhood $\Omega$ of $p$ such that $u_{1}=D u$ has a well defined trace $T_{\tau} u_{1}$ defined on the translates $(\tau N+\Sigma) \cap \Omega$ along $N$ satisfying the following properties:

(1) $\lim _{\varepsilon \backslash 0} T_{\tau+\varepsilon} u_{1}=T_{\tau} u_{1}$ (one-sided continuity);

(2) if $T_{0} u_{1}=0$ on $\Sigma \cap \Omega$ then $u$ vanishes identically on a neighborhood of $p$ (uniqueness).

Proof. We may find local $C^{1}$ coordinates $x_{1}, \ldots, x_{m}, t_{1}, \ldots, t_{n}$, defined in a neighborhood $\Omega$ of $p$ so that $x_{k}(p)=t_{j}(p)=0,1 \leq j \leq n, 1 \leq k \leq m$, and a complete set of first integrals is of the form

$$
Z_{j}(x, t)=x_{j}+i \varphi_{j}(x, t), \quad j=1, \ldots, m,
$$

with $\partial \varphi_{j} / \partial x_{k}(0,0)=0$. Furthermore, we may assume that $\Sigma \cap \Omega$ is given by $t_{1}=0$ and that $N$ points in the direction of increasing $t_{1}$. If $n=1$, we have just seen that taking $D=\operatorname{det} Z_{x}$ the conclusions of the theorem are obtained. For $n>1$ we discard the vector fields $L_{2}, \ldots, L_{n}$ and rename the coordinates as follows: $t^{\prime}=t_{1}$, $x_{k}^{\prime}=x_{k}, 1 \leq k \leq m, x_{m+k}^{\prime}=t_{k+1}, 1 \leq k \leq n-1$. Then we add new first integrals $Z_{m+1}\left(x^{\prime}, t^{\prime}\right)=x_{m+1}^{\prime}, \ldots, Z_{m+n-1}\left(x^{\prime}, t^{\prime}\right)=x_{m+n-1}^{\prime}$. This leads us to a new locally integrable structure $\tilde{\mathcal{L}}$ of dimension 1 spanned by $L=L_{1}$ such that $\tilde{\mathcal{L}} u=0$. Hence, we may reason as before and conclude that $\operatorname{det} Z_{x} u$ has a trace on $\Sigma$ with the required properties.

Note that we may as well define a trace that possesses the uniqueness property (2) but instead of (1) satisfies $\lim _{\varepsilon \backslash 0} T_{\tau-\varepsilon} u_{1}=T_{\tau} u_{1}$.

If $u$ vanishes as a distribution above a noncharacteristic hypersurface $\Sigma$ it is clear that $T_{\tau} u_{1}=0$ for $\tau>0$ and sufficiently small. Then the continuity property (1) of the trace $T_{\tau} u_{1}$ implies that $T_{0} u_{1}=0$, so the support of $u$ cannot meet $\Sigma$ by property (2). If $u$ vanishes below $\Sigma$ we reach the same conclusion by using the trace that is continuous from the left. It follows from Theorem 2.3 that the support of $u$ propagates along the normal of noncharacteristic hypersurfaces, which implies in a standard way that the support of $u$ is a union of orbits of $\mathcal{L}$.

COROLlary 2.4. Let $\mathcal{L}$ be a locally integrable continuous subbundle of $\mathbb{C} T(\Omega)$, $\Omega \subset \mathbb{R}^{N}$ open. Let $u \in \mathcal{D}_{0}^{\prime}(\Omega)$ be a Radon measure such that $\mathcal{L} u=0$. Then the support of $u$ is a union of orbits of $\mathcal{L}$ in $\Omega$.

For the case of a $\mathrm{CR}$ structure on a generic $\mathrm{CR}$ submanifold $M \subset \mathbb{C}^{n}$ of class $C^{1}$, we recover a result of $[\mathrm{CR}]$ :

Corollary 2.5. ([CR]) Let $M$ be a generic CR submanifold of $\mathbb{C}^{n}$ of class $C^{1}$. Then the support of a $C R$ distribution $u \in \mathcal{D}_{0}^{\prime}(M)$ is a union of orbits.

Remark 2.6. In the CR case, there are two differences between our results here and the ones proved in the paper [CR]. First, in [CR], Corollary 2.5 is deduced from a weaker uniqueness result (Lemma A.5 in [CR]) than the one stated in part 2 of Theorem 2.3. Second, the results in $[\mathrm{CR}]$ do not show that a measure solution can be approximated by $C^{1}$ solutions as is done in section 3 here (see Theorem 3.2). 
Remark 2.7. If $\mu$ is a Radon measure that has a trace defined at a family of translates of a hypersurface $\Sigma$ and $f$ is a continuous function, it is not true, in general, that the product $f \mu$ also has a trace. For instance, the function $\mu(x, t)=1 /(x+i t) \in$ $L_{\text {loc }}^{1}\left(\mathbb{R}^{2}\right)$ has a trace $T_{t} \mu$, continuous from the right, given by the locally integrable function $\mathbb{R} \ni x \mapsto 1 /(x+i t)$ for $t \neq 0$ and equal to the distribution $T_{0} \mu=\operatorname{pv}(1 / x)-i \pi \delta$ for $t=0$. Consider the continuous function defined by $f(x, t)=0$ for $x \leq 0$ and $f(x, t)=1 /|\ln x|$ for $x>0$ and set $\nu(x, t)=(f \mu)(x, t)$. Thus, $\nu \in L_{\text {loc }}^{1}\left(\mathbb{R}^{2}\right)$ is continuous off the origin and has a natural restriction to the straight lines $t=$ constant $\neq 0$ given by restriction. On the other hand, if $v(x) \in C_{c}^{\infty}(\mathbb{R})$ is real and $v(0) \neq 0$ we see that

$$
\lim _{t \rightarrow 0} \operatorname{Re} \int \nu(x, t) v(x) d x=\int_{0}^{\infty} \frac{v(x)}{x|\ln x|} d x=\infty,
$$

so it is not possible to define a one-sided continuous trace for $\nu$ that coincides with restriction for $t \neq 0$.

3. Approximation of measure solutions by smoother solutions.. Assume as in section 2 that $\mathcal{L}$ is a continuous locally integrable structure defined in an open subset $\Omega$ of $\mathbb{R}^{N}$ that contains the origin, with fiber dimension $n$, and that $u \in \mathcal{D}_{0}^{\prime}(\Omega)$ satisfies the equation $\mathcal{L} u=0$. Shrinking $\Omega$ we may assume that $u$ is a finite measure and choosing appropriate $C^{1}$ coordinates $x_{1}, \ldots, x_{m}, t_{1}, \ldots, t_{n}$, we may assume that a complete set of first integrals are of the form

$$
Z_{j}(x, t)=x_{j}+i \varphi_{j}(x, t), \quad j=1, \ldots, m,
$$

with $\varphi_{j}$ real satisfying $\partial \varphi_{j} / \partial x_{k}(0,0)=0$ and $\left|\partial \varphi_{j} / \partial x_{k}(0,0)\right|$ small throughout $\Omega$. Consider balls $B_{1} \subset \mathbb{R}^{m}, B_{2} \subset \mathbb{R}^{n}, B_{1}=B\left(0, r_{1}\right), B_{2}=B\left(0, r_{2}\right)$, such that $B_{1} \times B_{2} \subset$ $\Omega$, and for $\psi(x) \in C_{c}^{1}\left(B_{1}\right)$ set

$$
\left\langle\left(\operatorname{det} Z_{x} u\right)_{\psi}, \phi\right\rangle=\left\langle\operatorname{det} Z_{x} u, \psi \otimes \phi\right\rangle, \quad \phi(t) \in C_{c}^{1}\left(B_{2}\right) .
$$

If follows from the discussion in section 2 that $\left(\operatorname{det} Z_{x} u\right)_{\psi}$ is a finite measure on $B_{2}$ such that its exterior derivative $d_{t}\left(\operatorname{det} Z_{x} u\right)_{\psi}$ is also a finite measure. In other words, $\left(\operatorname{det} Z_{x} u\right)_{\psi}$ may be identified with a function of bounded variation $f_{\psi}(t)=$ $\langle f(t), \psi\rangle \in B V(W), \bar{B}_{2} \subset W$. We now recall some general properties of functions of bounded variation (see, e.g., [G]): if $g(t) \in B V(W)$ then $g(t) \in L^{1}(W)$ and its derivatives $\partial g / \partial t_{j}=\mu_{j}$ are measures with total bounded variation $\left\|\mu_{j}\right\|=\left|\mu_{j}\right|(W)$, where $|\mu|$ is the variation of $\mu$. In fact, $g(t)$ belongs to the smaller space $L^{p}\left(B_{2}\right)$, with $p=p(n)=n /(n-1)$, and the generalized Poincaré inequality holds: if we denote by

$$
g_{B_{2}}=\frac{1}{\left|B_{2}\right|} \int_{B_{2}} g(t) d t
$$

the mean value of $g$ on $B_{2}$, then

$$
\left\|g-g_{B_{2}}\right\|_{L^{p}\left(B_{2}\right)} \leq C \sum_{j=1}^{n}\left|\mu_{j}\right|\left(B_{2}\right)
$$

where $C>0$ depends on the dimension $n$ but neither on the radius of $B_{2}$ nor on $g$. A pointwise estimate related to the Poincaré inequality is given in the following lemma. 
Lemma 3.1. Let $g$ be of bounded variation on an open set $W \subset \mathbb{R}^{n}$ that contains $\bar{B}_{2}$ and set $\partial g / \partial t_{j}=\mu_{j},|\mu|=\sum_{j=1}^{n}\left|\mu_{j}\right|$. Then

$$
\left|g(t)-g_{B_{2}}\right| \leq C \int_{B_{2}}|t-s|^{1-n} d|\mu|(s), \quad \text { a.e. } t \in B_{2}
$$

where $C$ depends only on $n$.

Proof. For $g \in W^{1,1}\left(B_{2}\right)$ the lemma is well known (see, e.g., [GT,Lemma 7.16]). Let $g$ be as in the lemma. Let $\eta(s) \in C_{c}^{\infty}\left(\mathbb{R}^{n}\right)$ such that $\eta(s)$ is nonnegative, its support lies in the unit ball $B_{1}(0)$ and $\int_{\mathbb{R}^{n}} \eta(s) d s=1$. Set $\eta_{\epsilon}(s)=\epsilon^{-n} \eta(s / \epsilon)$. If

$$
g_{\epsilon}(t)=\eta_{\epsilon} * g(t),
$$

then $g_{\epsilon} \rightarrow g$ in $L^{1}$ and $g_{\epsilon}(t) \rightarrow g(t)$ a.e. For $t \in B_{2}$, and for any $\epsilon>0$, we have

$$
\left|g_{\epsilon}(t)-\left(g_{\epsilon}\right)_{B_{2}}\right| \leq C \int_{B_{2}} \frac{\left|D g_{\epsilon}(s)\right|}{|s-t|^{n-1}} d s .
$$

Note that

$$
D g_{\epsilon}(s)=\eta_{\epsilon} * \mu(s)
$$

where $\mu=\left(\mu_{1}, \ldots, \mu_{n}\right)$. It follows from (3.2) that

$$
\left|g_{\epsilon}(t)-\left(g_{\epsilon}\right)_{B_{2}}\right| \leq C \int_{B_{r_{2}+\epsilon}}\left(\int_{B_{2}} \frac{\eta_{\epsilon}\left(s^{\prime}-s\right)}{|s-t|^{n-1}} d s\right) d|\mu|\left(s^{\prime}\right) .
$$

We will estimate the inner integral which after a change of variables is dominated by

$$
\int_{|s| \leq 1} \frac{1}{\left|s^{\prime}-t-\epsilon s\right|^{n-1}} d s .
$$

For $y \in \mathbb{R}^{n}$ fixed, $y \neq 0$, consider therefore the integral

$$
\int_{|s| \leq 1} \frac{1}{|y-\epsilon s|^{n-1}} d s .
$$

If $|y| \geq 2 \epsilon$, then for all $|s| \leq 1,|y-\epsilon s| \geq \frac{|y|}{2}$ and so for such $y$,

$$
\int_{|s| \leq 1} \frac{1}{|y-\epsilon s|^{n-1}} d s \leq \frac{c_{n}}{|y|^{n-1}} .
$$

Suppose $|y| \leq 2 \epsilon$. In the integral in (3.4), change variables $t^{\prime}=\epsilon s$. We then get

$$
\begin{aligned}
\int_{|s| \leq 1} \frac{1}{|y-\epsilon s|^{n-1}} d s & =\frac{1}{\epsilon^{n}} \int_{\left|t^{\prime}\right| \leq \epsilon} \frac{1}{\left|y-t^{\prime}\right|^{n-1}} d t^{\prime} \\
& \leq \frac{1}{\epsilon^{n}} \int_{\left|t^{\prime}\right| \leq 3 \epsilon} \frac{1}{\left|t^{\prime}\right|^{n-1}} d t^{\prime} \\
& \leq \frac{c}{\epsilon^{n-1}} \leq \frac{c_{n}}{|y|^{n-1}} .
\end{aligned}
$$

It now follows that for some dimensional constant $C_{n}$,

$$
\int_{B_{2}} \frac{\eta_{\epsilon}\left(s^{\prime}-s\right)}{|s-t|^{n-1}} d s \leq \int_{|s| \leq 1} \frac{1}{\left|s^{\prime}-t-\epsilon s\right|^{n-1}} d s \leq \frac{C_{n}}{\left|s^{\prime}-t\right|^{n-1}} .
$$


To prove (3.1) for a fixed $t \in B_{2}$, we may assume - and we will do so- that the function $s \mapsto|t-s|^{1-n} \in L^{1}\left(B_{2}, d|\mu|\right)$, otherwise the right hand side would be infinity and the inequality trivial. By the Dominated Convergence Theorem, it follows that

$$
\begin{aligned}
\lim _{\epsilon \rightarrow 0} \int_{B_{r_{2}+\epsilon}}\left(\int_{B_{2}} \frac{\eta_{\epsilon}\left(s^{\prime}-s\right)}{|s-t|^{n-1}} d s\right) & d|\mu|\left(s^{\prime}\right) \\
& =\int_{s^{\prime}} \lim _{\epsilon \rightarrow 0} \chi_{B_{r_{2}+\epsilon}}\left(s^{\prime}\right)\left(\int_{B_{2}} \frac{\eta_{\epsilon}\left(s^{\prime}-s\right)}{|s-t|^{n-1}} d s\right) d|\mu|\left(s^{\prime}\right) \\
& =\int_{\bar{B}_{2}}\left(\int_{t^{\prime}} \frac{\eta\left(t^{\prime}\right)}{\left|s^{\prime}-t\right|^{n-1}} d t^{\prime}\right) d|\mu|\left(s^{\prime}\right) \\
& =\int_{\bar{B}_{2}} \frac{1}{\left|s^{\prime}-t\right|^{n-1}} d|\mu|\left(s^{\prime}\right)
\end{aligned}
$$

where $\chi_{B_{r_{2}+\epsilon}}\left(s^{\prime}\right)$ denotes the characteristic function of the ball $B_{r_{2}+\epsilon}$. Thus, letting $\epsilon \searrow 0$ in (3.3), we obtain estimate (3.1) with the integral on the right hand side taken over $\bar{B}_{2}$ instead of $B_{2}$. But then we obtain (3.1) by expressing $B_{2}=B\left(0, r_{2}\right)$ as an increasing countable union of closed balls of radius $<r_{2}$ and applying the weaker estimate to the smaller closed balls. This proves the lemma.

Remark. It is known that (3.2) holds with $C=\gamma_{n}=2^{n-1} \Gamma(n / 2) / \pi^{n / 2}$. Then the trivial estimate $\left|D g_{\epsilon}(s)\right| \leq \sum_{j=1}^{n}\left|D_{j} g_{\epsilon}(s)\right|$ implies that we may take $C=\gamma_{n}$ in (3.3) and the proof of the lemma shows that (3.1) is valid with $C=\gamma_{n}$.

We will now introduce a variation of the Baouendi-Treves approximation formula that does not require a continuous trace of the solution to maximally real submanifolds. For $(x, t) \in B_{1} \times B_{2}, \tau \geq 1$, and a fixed bump function $h(x) \in C_{c}^{\infty}\left(B_{1}\right)$ that is identically 1 on a neighborhood of the origin, set

$$
F_{\tau} u(x, t)=(\tau / \pi)^{m / 2} \frac{1}{\left|B_{2}\right|} \int_{\mathbb{R}^{m} \times B_{2}} e^{-\tau[Z(x, t)-Z(y, s)]^{2}} \operatorname{det} Z_{y}(y, s) u(y, s) h(y) d y d s .
$$

The integral

$$
\int_{\mathbb{R}^{m} \times B_{2}} e^{-\tau[Z(x, t)-Z(y, s)]^{2}} \operatorname{det} Z_{y}(y, s) u(y, s) h(y) d y d s
$$

in the definition of $F_{\tau} u(x, t)$ is understood as

$$
\int_{B_{2}}\left\langle f(s), e^{-\tau[Z(x, t)-Z(\cdot, s)]^{2}} h(\cdot)\right\rangle d s .
$$

It is clear that, for fixed $\tau \geq 1, F_{\tau} u(x, t)$ is an entire function of $Z(x, t)$, in particular, it satisfies the equation $\mathcal{L} E_{\tau} u=0$ in the classical sense and is of class $C^{1}$. We wish to show that, for $r_{2}$ sufficiently small, $F_{\tau} u(x, t) \rightarrow u(x, t)$ in the weak-* topology of $C_{c}^{0}(U)^{*}$ as $\tau \rightarrow \infty$ for a convenient neighborhood of the origin $U \subset \mathbb{R}^{m} \times \mathbb{R}^{n}$. More precisely, we will show that, given $v \in C_{c}^{0}(U)$,

$$
\left\langle u-F_{\tau} u, v\right\rangle \longrightarrow 0 \quad \text { as } \quad \tau \rightarrow \infty .
$$

For $s \in B_{2}$ and $\zeta \in \mathbb{C}^{m}$ consider the function

$$
g^{\tau}(s, \zeta)=(\tau / \pi)^{m / 2} \int_{\mathbb{R}^{m}} e^{-\tau[\zeta-Z(y, s)]^{2}} \operatorname{det} Z_{y}(y, s) u(y, s) h(y) d y .
$$


Notice that $g^{\tau}(s, \zeta)$ is of the form $\left(\operatorname{det} Z_{x} u\right)_{\psi}$ for a convenient $\psi$ depending on $\tau$ and $\zeta$ so $g^{\tau}(s, \zeta)$ is defined for all $\tau \geq 1$, all $\zeta \in \mathbb{C}$ and all $s \in B_{2} \backslash E$, with $|E|=0$, and belongs to $L^{p}\left(B_{2}\right)$ for $\tau, \zeta$ fixed. To see that $E$ can be taken independently of $\zeta$ and $\tau$, we may replace the exponential by its Taylor series, obtaining a power series in $\zeta$ and $\tau$ with coefficients that are linear combinations of functions of the form

$$
c_{\alpha}(s)=\int_{\mathbb{R}^{m}} Z(y, s)^{\alpha} \operatorname{det} Z_{y}(y, s) u(y, s) h(y) d y, \quad \alpha \in \mathbb{Z}_{+}^{n},
$$

defined for $s \in B_{2} \backslash E_{\alpha},\left|E_{\alpha}\right|=0$. Then we take $E=\bigcup_{\alpha} E_{\alpha}$. The norm of $c_{\alpha}(s)$ in $B V\left(B_{2}\right)$ is dominated by the $C^{1}$ norm of $Z(y, s)^{\alpha} h(y)$, which has polynomial growth in $\alpha$, so the argument shows that we may regard $(\zeta, \tau) \mapsto g^{\tau}(s, \zeta)$ as an entire holomorphic function with values in $B V\left(B_{2}\right)$. Using (3.1) we get

$$
\left|g^{\tau}(t, \zeta)-g_{B_{2}}^{\tau}(\zeta)\right| \leq C \int_{B_{2}}|t-s|^{1-n} d\left|\mu^{\tau}\right|(s, \zeta), \quad \text { a.e. } t \in B_{2} .
$$

For $\zeta=Z(x, t)$ we get

$$
\begin{aligned}
g^{\tau}(t, Z(x, t)) & =G_{\tau} u(x, t) \\
g_{B_{2}}^{\tau}(Z(x, t)) & =F_{\tau} u(x, t)
\end{aligned}
$$

so (3.6) yields

$$
\left|G_{\tau} u(x, t)-F_{\tau} u(x, t)\right| \leq C \int_{B_{2}}|t-s|^{1-n} d\left|\mu^{\tau}\right|(s, Z(x, t)),
$$

for a.e. $t \in B_{2}$. To take advantage of estimate (3.7) we need to compute

$$
\begin{aligned}
\mu_{j}^{\tau}(t, \zeta) & =\frac{\partial}{\partial t_{j}} g^{\tau}(t, \zeta) \\
& =\frac{\partial}{\partial t_{j}} \int_{\mathbb{R}^{m}} e^{-\tau[\zeta-Z(y, t)]^{2}} \operatorname{det} Z_{x}(y, t) u(y, t) h(y) d y
\end{aligned}
$$

Differentiating under the integral sign, using the equation $L_{j} u=0$ in order to replace $\partial_{t_{j}}\left(\operatorname{det} Z_{x} u\right)$ by $-\sum_{k=1}^{m} \partial_{y_{k}}\left(\operatorname{det} Z_{x} \lambda_{j k} u\right)$ in the first term on the right, integrating by parts with respect to $y$ and using the fact that

$$
\left(\partial_{t_{j}}+\sum_{k=1}^{m} \lambda_{j k} \partial_{y_{k}}\right) e^{-\tau[\zeta-Z(y, t)]^{2}}=0
$$

we get

$$
\begin{aligned}
\mu_{j}^{\tau}(t, \zeta) & =\int_{\mathbb{R}^{m}} e^{-\tau[\zeta-Z(y, t)]^{2}}\left(\operatorname{det} Z_{x} u\right)(y, t) \sum_{k=1}^{m} \lambda_{j k} \frac{\partial h(y)}{\partial y_{k}} d y . \\
& =\int_{\mathbb{R}^{m}} e^{-\tau[\zeta-Z(y, t)]^{2}}\left(\operatorname{det} Z_{x} u\right)(y, t) h_{j}(y, t) d y .
\end{aligned}
$$

To estimate the variation $\left|\mu_{j}^{\tau}(\zeta)\right|$ of $\mu_{j}^{\tau}(\cdot, \zeta)$ on $B_{2}$ observe that if $\phi(t) \in L^{\infty}\left(B_{2}\right)$,

$$
\int \mu_{j}^{\tau}(t, \zeta) \phi(t) d t=\int e^{-\tau[\zeta-Z(y, t)]^{2}} \operatorname{det} Z_{x}(y, t) h_{j}(y, t) \phi(t) d u(y, t)
$$


so, for any Borel set $E \subset B_{2}$,

$$
\int_{E} d\left|\mu_{j}^{\tau}\right|(\zeta) \leq C \sup _{(y, s) \in \operatorname{supp} d h \times B_{2}} e^{-\tau \Re[\zeta-Z(y, s)]^{2}} \int_{B_{1} \times E} d|u|
$$

where $|u|$ denotes the variation of $u$. For $\zeta=Z(x, t)$ we get

$$
\int_{E} d\left|\mu_{j}^{\tau}\right|(Z(x, t)) \leq C \sup _{\left\{2 \delta \leq|y|<r_{1}\right\} \times B_{2}} e^{-\tau \Re[Z(x, t)-Z(y, s)]^{2}} \int_{B_{1} \times E} d|u|
$$

if we assume that $h(y)$ has been chosen to satisfy $h(y) \equiv 1$ for $|y| \leq 2 \delta<r_{1}$. Let $v(x, t) \in C_{c}^{0}\left(B(0, \delta) \times B_{2}\right)$ and consider

$$
I_{\tau}(v)=\int\left(G_{\tau} u(x, t)-F_{\tau} u(x, t)\right) v(x, t) d x d t .
$$

If $(x, t)$ is in the support of $v(x, t)$ and $r_{2}$ is chosen conveniently small (depending on $\delta$ ) we verify that, in the exponent on the right hand side of $(3.8), \Re[Z(x, t)-Z(y, s)]^{2} \geq$ $c>0$. Thus, in view of (3.7) and (3.8),

$$
\begin{aligned}
\left|I_{\tau}(v)\right| & \leq C\|v\|_{L^{\infty}} e^{-c \tau} \int_{B_{1} \times B_{2}} \int_{B_{1} \times B_{2}} \frac{d x d t}{|t-s|^{n-1}} d|u|(y, s) \\
& \leq C\|v\|_{L^{\infty}}|u|\left(B_{1} \times B_{2}\right) e^{-c \tau} .
\end{aligned}
$$

This shows that $I_{\tau}(v) \rightarrow 0$ as $\tau \rightarrow \infty$. On the other hand, adapting the arguments in $[\mathrm{HM}]$ (where $Z(x, t)$ is smooth) there is no difficulty in proving that, since $u(x, t)$ is a bounded measure, there is a neighborhood $U$ of the origin in $\mathbb{R}^{m} \times \mathbb{R}^{n}$ such that $G_{\tau} u \rightarrow u$ in the weak-* topology of $C_{c}^{0}(U)^{*}$. Thus,

$$
\left\langle u-F_{\tau} u, v\right\rangle \longrightarrow 0 \quad \text { as } \tau \rightarrow \infty,
$$

as we wished to prove.

Remark. Integrating (3.7) with respect to $d x d t$ on $B_{1} \times B_{2}$ and taking account of (3.8) we see that

$$
\left\|G_{\tau} u-F_{\tau}\right\|_{L^{1}(U)} \leq C, \quad \tau \geq 1 .
$$

Since, it is known that $\left\|G_{\tau} u\right\|_{L^{1}(U)} \leq C$ it follows that $\left\|F_{\tau} u\right\|_{L^{1}(U)} \leq C$ as well.

We have proved:

TheOREM 3.2. Let $\mathcal{L}$ be a locally integrable continuous subbundle of $\mathbb{C} T(\mathcal{M})$. Let $u \in \mathcal{D}_{0}^{\prime}$ be a Radon measure such that $\mathcal{L} u=0$. Then for each $p \in \mathcal{M}$, there is a neighborhood $\Omega$ and a sequence $P_{k}$ of holomorphic polynomials such that $u=$ $\lim _{k \rightarrow \infty} P_{k}(Z)$ in the weak-* topology of $C_{c}^{0}(\Omega)^{*}$ where $Z=\left(Z_{1}, \ldots, Z_{m}\right)$ is a complete set of first integrals on $\Omega$.

\section{REFERENCES}

[BT] M. S. Baouendi And F. Treves, A property of the functions and distributions annihilated by a locally integrable system of complex vector fields, Ann. of Math., 113 (1981), pp. 387421.

[CR] E. M. ChiRka And C. REA, Differentiable CR mappings and CR orbits, Duke Math. J., 94 (1998), pp. 325-340. 
[GT] D. Gilbard and N. Trudinger, Elliptic Partial Differential Equations of Second Order, Springer-Verlag Berlin Heidelberg New York, 2001.

[G] E. Giusti, Minimal surfaces and functions of bounded variation, Birkhäuser Boston, 1984.

[HM] J. Hounie And P. Malagutti, On the convergence of the Baouendi-Treves approximation formula, Comm. P.D.E., 23 (1998), pp. 1305-1347.

[T] F. Treves, Hypo-analytic structures, local theory, Princeton University Press, 1992. 